\title{
An Interesting Case of Heart Failure in a Patient with Bicuspid Aortic Valve - Unveiling the Diagnosis of Amyloidosis
}

\author{
Praveen N, O Adikesava Naidu, Anjani Kiranmayi K, Ravi Srinivas, KMK Reddy P \\ Department of Cardiology, Osmania General Hospital, Hyderabad
}

Correspondence should be addressed to Praveen Nagula, drpraveennagula@gmail.com

Received Date: May 31, 2020; Accepted Date: June 7, 2020; Published Date: June 14, 2020

\begin{abstract}
Restrictive cardiomyopathy (RCMP) is infrequent in occurrence compared to the other cardiomyopathies. The stiff ventricular walls impair the diastolic function, increase the filling pressures, and thereby result in heart failure. Amyloidosis, a systemic disorder is the most common cause of RCMP. The amyloid diagnosis is often missed as the features are masqueraded by the presence of other conditions. The Bicuspid aortic valve (BAV) is the most common congenital anomaly of the cardiovascular system occurring in approximately $0.5-2 \%$ of the general population. We present a case of bicuspid aortic valvular heart disease masking the amyloidosis with heart failure in a middle-aged male.
\end{abstract}

\section{KEYWORDS}

Restrictive cardiomyopathy; Amyloidosis; Bicuspid aortic valve; Heart failure

\section{CASE PRESENTATION}

A 45-year-old male, field worker, presented with complaints of swelling of lower limbs progressed to all over the abdomen leading to abdominal distention over six months. He had shortness of breath on exertion, progressing from NYHA class II to NYHA class III over two months. He was referred to us for further management as there was no improvement in his symptoms.

He had a history of jaundice three years before the current admission. During the evaluation of jaundice, he was diagnosed as having rheumatic heart disease, atrial fibrillation with heart failure. He was medically managed and was advised penicillin prophylaxis. Subsequently, with the improvement of symptoms, he used the medication for a year. He was noncompliant thereafter. He was a reformed smoker and an occasional alcoholic. On further evaluation, there was no history of coronary artery disease, hypertension, diabetes mellitus, thyroid disorder, anemia, or blood transfusions previously. His medications included digoxin $(0.5 \mathrm{mg} /$ day $)$, furosemide (20 mg twice daily), enalapril (5 $\mathrm{mg}$ twice daily), and vitamin supplements, which he was using for the past three months.

On examination, he was conscious, emaciated with bilateral suffused conjunctivae and anasarca. He had a raised jugular venous pulse (JVP) with a column height of $12 \mathrm{~cm}$ from the sternal angle. Prominent $\mathrm{v}$ waves were seen. He was afebrile. He had a low volume pulse with a rate of 58 per minute. His blood pressure recorded 90/50 
$\mathrm{mm}$ hg over the upper arm. There was no postural drop in blood pressure.

The cardiovascular examination revealed a down and out Apex of the left ventricular type in $6^{\text {th }}$ intercostal space with a heaving character. There was grade 2 parasternal heave. The S1 and S2 were normal. The S3, S4, and an ejection click were prominent. There was an early diastolic murmur occupying approximately $50 \%$ of diastole and a mid systolic murmur of grade $3 / 6$ in the aortic area. There was a dull note on percussion with decreased breath sounds bilaterally over the basal areas. There were moderate ascites with no apparent hepatomegaly. The veins over the abdomen were distended with venous filling from above downward. There was no neurological deficit.
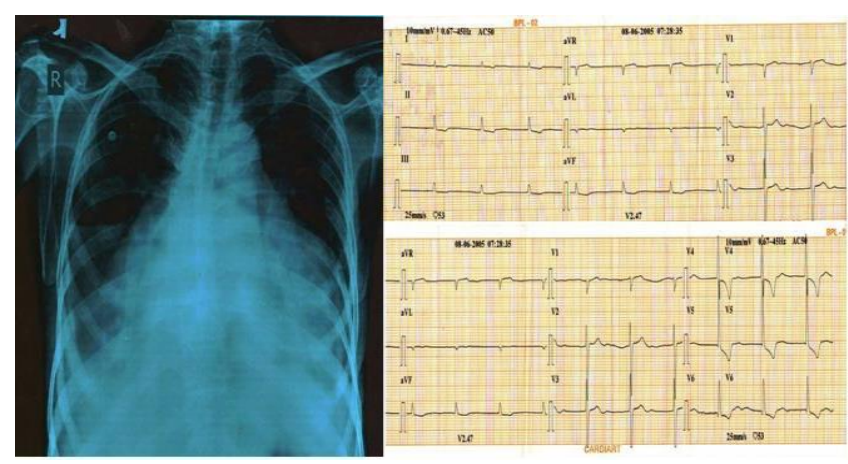

Figure 1: (Left) Chest X-ray posteroanterior (PA) view showing massive cardiomegaly, bilateral pleural effusion. (Right) The electrocardiogram showing junctional rhythm, right axis deviation of $+120^{\circ}$, left ventricular hypertrophy with strain pattern.

His biochemical profile showed hemoglobin of $11 \mathrm{gm} / \mathrm{dl}$, normal total leukocyte count, and normal differential cell count. The absolute eosinophil count was 330 cell/cumm. The total proteins were $10 \mathrm{gm} / \mathrm{dl}$ and had an albumin fraction of $6.5 \mathrm{gm} / \mathrm{dl}$. The complete urine examination showed traces of albumin. The usual viral screening was negative. The serum creatinine was $1.2 \mathrm{mg} / \mathrm{dl}$ with a blood urea of $34 \mathrm{mg} / \mathrm{dl}$. The serum electrolytes profile showed a a low potassium level of $2.8 \mathrm{mg} / \mathrm{dl}$ with normal sodium, chloride, and calcium levels. The troponin I was positive (qualitatively) with a creatine kinase (CK) level of 498
$\mathrm{IU} / \mathrm{ml}$ and creatine kinase myocardial brain fraction (CKMB) of $86 \mathrm{IU} / \mathrm{ml}$. The serum digoxin levels were not done.

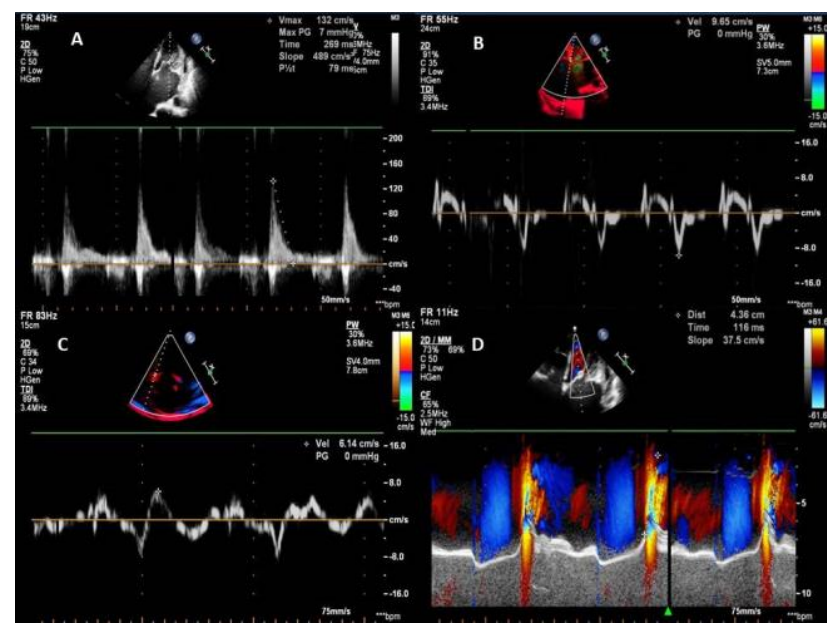

Figure 2: (A) Mitral inflow velocities showing early $E$ wave of $132 \mathrm{~cm} / \mathrm{sec}$ and absent A wave. (B and C) The tissue Doppler velocity of the lateral and medial mitral annulus with velocities of $9.2 \mathrm{~cm} / \mathrm{sec}$ and $8.5 \mathrm{~cm} / \mathrm{sec}$ respectively. (D) The color M mode of the mitral inflow showing the propagation velocity $(\mathrm{Vp})$ of $37.5 \mathrm{~cm} / \mathrm{sec}$.

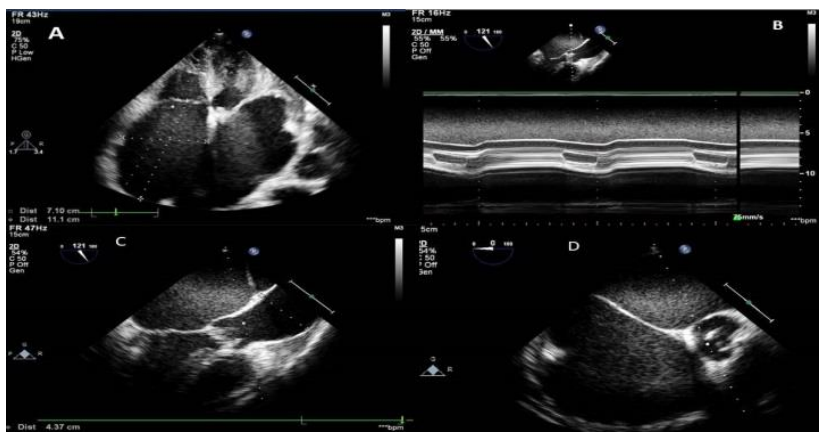

Figure 3: (A) Apical 4 chamber view showing biatrial enlargement. (B) The $\mathrm{M}$ mode of the aortic valve showing a positive eccentricity index with a deviation of aortic closure line to the lower line rather than being in the middle. (C) The ascending aorta on transesophageal echocardiogram mid esophageal level at 120 measured $4.3 \mathrm{~cm}$ in diameter (D)

Transesophageal echo view at midesophageal level 0 degree showing the bicuspid aortic valve with calcification, pseudoraphe oriented towards interatrial septum indicating fusion of right and noncoronary.

The chest $\mathrm{x}$-ray showed bilateral pleural effusion with right more than the left and massive cardiomegaly (Figure 1, left). The electrocardiogram (ECG) showed junctional rhythm with a heart rate of 56 per minute, poor $\mathrm{R}$ wave Progression. U waves were seen in the precordial leads from V3-V5 (Figure1, right). The mitral valve Doppler E/e' (averaged pulsed wave - Doppler to tissue Doppler of 
both lateral and medial annulus velocities) was 15.5 (Figure 2A - Figure 2C). The lateral mitral annular plane systolic velocity (s') was recorded as $6.5 \mathrm{~cm} / \mathrm{sec}$ (Figure 2C). The color M mode across the mitral valve showed propagation velocity $(\mathrm{Vp})$ of $37.5 \mathrm{~cm} / \mathrm{sec}$ (Figure 2D). There was massive biatrial enlargement (right atrium measuring $7.1 \mathrm{~cm} \times 11 \mathrm{~cm}$ and left atrium measuring 6.5 $\mathrm{cm} \times 9 \mathrm{~cm}$ ) with spontaneous echo contrast in the apical four-chamber view (Figure 3A). The interatrial septum was $3 \mathrm{~mm}$ in thickness. The aortic valve on $\mathrm{M}$ mode showed a positive eccentricity index (Figure 3B). The ascending aorta (AA) was dilated with a diameter of 4.37 $\mathrm{cm}$ (Figure 3C) with an annulus measurement of $2.2 \mathrm{~cm}$. There was a fusion of the right and noncoronary cusps when assessed at the great artery level with the opening of the aortic valve in right and left orientation (Figure 3D).

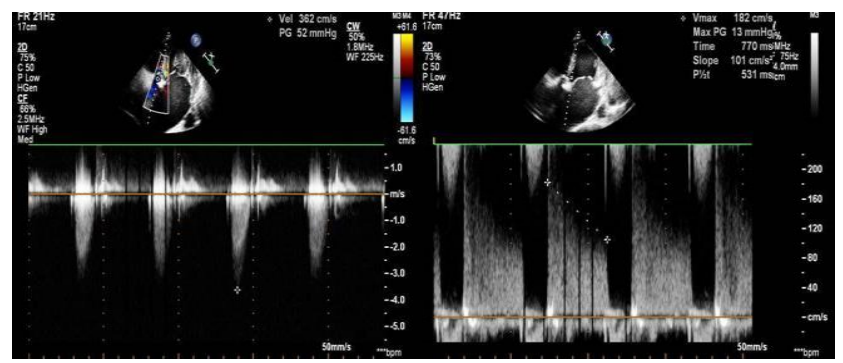

Figure 4: (Left) Continuous-wave Doppler of the aortic valve showing a peak velocity of $3.62 \mathrm{~m} / \mathrm{sec}$ and peak pressure gradient of $52 \mathrm{mmHg}$ with a mean of $32 \mathrm{mmHg}$. (Right) Aortic regurgitation pressure half time of $530 \mathrm{msec}$.

The continuous wave Doppler (CWD) across the aortic valve recorded a peak velocity of $3.62 \mathrm{~m} / \mathrm{sec}$ with peak pressure gradient of $52 \mathrm{~mm} \mathrm{Hg}$ and a mean of $32 \mathrm{~mm} \mathrm{Hg}$ (Figure 4, left). There was mild aortic regurgitation based on pressure half time of $530 \mathrm{msec}$ (Figure 4, right). There was moderate eccentric mitral regurgitation with tethering of the posterior mitral leaflet (Figure 5, upper left). The mitral leaflet thickness measured $3.4 \mathrm{~mm}$ in mid diastole. The stroke volume and assessment of the aortic valve area by the continuity equation was not calculated because of the presence of aortic regurgitation and the mitral regurgitation. The left ventricular function was normal. The relative wall thickness was 0.23 and the end-diastolic dimension of the left ventricle (LVEDD) was $50 \mathrm{~mm}$ (Figure 5, upper right). The tricuspid regurgitation (TR) on assessment had a peak velocity of $2.9 \mathrm{~m} / \mathrm{sec}$ and the systolic pressure of the right ventricle was measured as 54 $\mathrm{mmHg}$ (Figure 5, lower left). The inferior vena cava was dilated with $2.5 \mathrm{~cm}$ diameter and collapsing less than $50 \%$ with respiration (Figure 5, lower right). The tricuspid annular plane systolic excursion (TAPSE) was $1.5 \mathrm{~cm}$ and the systolic annular plane velocity (TASPV) was $8.7 \mathrm{~cm} / \mathrm{sec}$. A diagnosis of atrial fibrillation, bicuspid aortic valve disease, paradoxical low flow low gradient severe aortic stenosis $(\mathrm{MG}<40 \mathrm{~mm}$ hg, Normal LVEF) with mild aortic regurgitation, moderate mitral regurgitation, normal left ventricular function, mild right ventricular dysfunction, moderate pulmonary hypertension, massive biatrial enlargement with spontaneous echo contrast and a dilated ascending aorta was made.

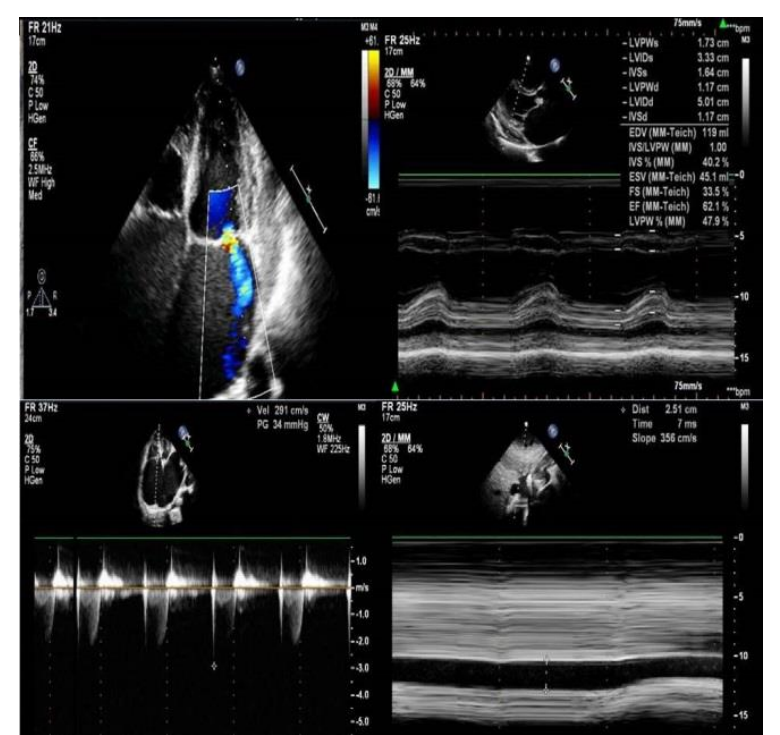

Figure 5: (Upper left) Apical 4 chamber view showing mitral regurgitation. (Upper right) M mode showing normal Left ventricular function with a left ventricular internal diastolic diameter of $50 \mathrm{~mm}$ and a relative wall thickness of 0.23. (Lower left) Tricuspid flow velocities measuring $2.9 \mathrm{~m} / \mathrm{sec}$ (Lower right) inferior vena cava measuring $2.5 \mathrm{~cm}$ with less than $50 \%$ collapse on respiration. 


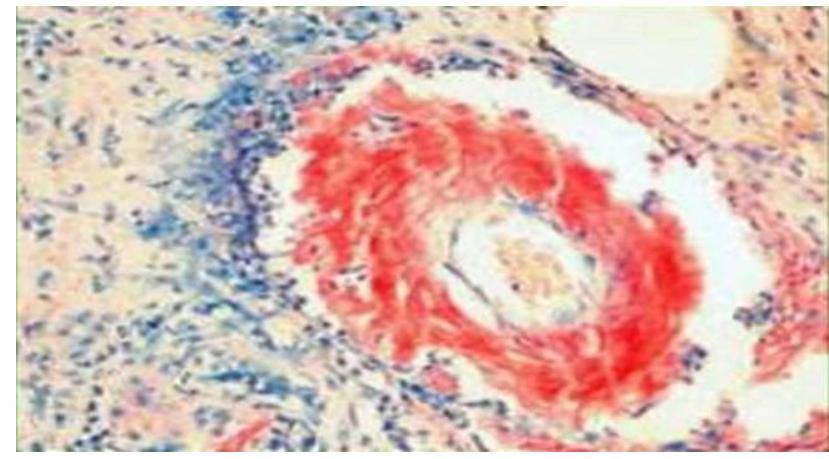

Figure 6: Congo red stain of the amyloid infiltrate from the abdominal fat pad biopsy.

The ultrasound of the abdomen showed ascites, mild hepatomegaly, normal gallbladder, and pancreas with no focal lesions. There was bilateral grade I renal parenchymal disease.

The computed tomography of the chest and abdomen showed massive cardiomegaly, bilateral pleural effusion, ascites, and bilateral renal parenchymal disease. There was no hilar, paraaortic, and retro aortic lymphadenopathy. The abdominal fat pad biopsy was done because of the discrepancy of symptoms, clinical examination, and the features on the echocardiogram, not fitting into a single diagnosis of valvular heart disease leading to heart failure. The congo red stain was positive for amyloid in the biopsy (Figure 6). Further analysis of the type of amyloidosis was not done because of financial constraints and his clinical status.

The digoxin was stopped because of the junctional escape rhythm and atrial fibrillation. Potassium supplementation was given. He was kept on an optimal dose of diuretics along with heart failure management as per the guidelines. He improved symptomatically.

After a year, when enquired it was known that he had sudden cardiac death while walking in his fields.

\section{DISCUSSION}

Restrictive cardiomyopathy (RCMP), the infrequent one among all the cardiomyopathies presents with diastolic heart failure. The most common cause is amyloid infiltration. Amyloidosis is a systemic disorder with the most common age of presentation being in the sixth decade. It can be light chain (AL), senile, or transthyretin (ATTR) amyloidosis. The diagnosis is mostly by exclusion [1]. Bicuspid aortic valve is the commonest cardiovascular abnormality occurring in approximately $2 \%$ of population [2]. It usually asymptomatic but can manifest as either stenosis or regurgitation or both secondary to increased wear and tear of the leaflets.

The aortic stenosis and restrictive cardiomyopathy both lead to ventricular hypertrophy and diastolic dysfunction. The biatrial enlargement and pulmonary hypertension in an isolated aortic valve disease are relatively late in onset unless concomitant mitral valve disease and atrial fibrillation are present. The other differential diagnosis for biatrial enlargement would be restrictive cardiomyopathy, constrictive pericarditis and idiopathic dilation of the atria (atrial cardiomyopathy) [3,4].

We suspected cardiac amyloidosis because of his (A) heart failure symptoms disproportionate to the echocardiographic findings of aortic stenosis (B) The presence of atrial fibrillation with junctional escape rhythm on use of digoxin.

Diagnosis of amyloidosis requires tissue biopsy positive for the amyloid on congo red stain and having apple birefringence when seen under polaroid microscopy. The most preferred site for the biopsy is either an abdominal fat pad or a rectal fat pad. Though they are most preferred because of ease of access, they are not specific. The specific site would be the organ suspected (here it is endomyocardial biopsy). The Fat pad biopsy indicating amyloid infiltration along with echo features of diastolic dysfunction makes the need for endomyocardial biopsy unlikely in this case. The positive congo red stain is specific for the presence of amyloid protein in a tissue [5]. 
There is increased susceptibility to digoxin toxicity on its use secondary to accumulation of the drug as a result of increased binding of the drug to amyloid protein [6]. The beta-blockers and angiotensin-converting enzyme inhibitors should be used cautiously though they have a mortality benefit in other scenarios of heart failure [6]. There is increased susceptibility to thrombosis with biatrial enlargement [5,7]. With increased imaging modalities (Cardiac MRI with tissue characterization) there is increased documentation of presence of amyloidosis in patients with severe aortic stenosis. The patients with both amyloidosis and aortic stenosis, benefit from percutaneous valve replacement compared to surgical valve replacement, as the latter is associated with complications in presence of amyloidosis [8]. Cardiac Amyloidosis has a poor prognosis after the onset of heart failure [5]. Sudden cardiac death is common in amyloidosis secondary to either severe heart failure, a progressive conduction disease, or severe valvular stenosis.

\section{LEARNING POINTS}

1. Amyloidosis should be suspected in a patient with heart failure not correlating with the severity of aortic stenosis.

2. Amyloidosis can lead to complete heart block in an atrial fibrillation patient when digoxin is used secondary to increased binding of the drug to protein (increased susceptibility to digoxin toxicity on regular doses).

3. The severity of aortic stenosis (AS) can be underestimated due to preserved left ventricular function and low gradients (paradoxical low flow low gradient AS) and hence they need additional imaging techniques for assessment of severity of AS (CT aortic calcium).

4. Amyloidosis is being diagnosed in increased frequencies in patients with aortic stenosis as a result of improved and advanced imaging modalities (Cardiac MRI with tissue characterization). When diagnosed, It can help in risk stratification and management strategy of the patients.

5. Patients with Amyloidosis and aortic stenosis should be managed early by percutaneous transcatheter valve replacement rather than surgery.

\section{CONCLUSION}

Heart failure due to amyloidosis can be masked by other conditions, such as aortic stenosis and mitral regurgitation in this case and high index of suspicion is needed. Additional imaging techniques are required for diagnosis and risk stratification.

\section{ACKNOWLEDGEMENT}

Dr. Srinivas, Associate Professor of Pathology, presently working in Gandhi Medical College, Hyderabad for the abdominal fat pad biopsy and congored stain of the biopsy.

The authors acknowledge the support of nursing staff of ICCU, male cardiology ward, technicians of echo room and the staff of Radiology and Pathology department, Osmania General Hospital, Hyderabad..

\section{CONTRIBUTION OF AUTHORS}

All authors have done the literature review and drafted the manuscript. The drafted manuscript was edited by the corresponding author. All the authors have read the final manuscript and approved it before final submission by the corresponding author to the journal.

\section{CONFLICTS OF INTEREST}

The authors state that they have no conflicts of interest to report. 


\section{REFERENCES}

1. Rodney HF, Ray EH (2018) The Dilated, restrictive, and infiltrative cardiomyopathy (11th edn.), Braunwald's Heart Disease: A Textbook of Cardiovascular Medicine, Elsevier Inc., Philadelphia, PA 1591.

2. Bicuspid aortic valve disease (2014) (11th edn.). Braunwald's Heart Disease: A Textbook of Cardiovascular Medicine, Elsevier Inc., Philadelphia, PA 1410.

3. Iqbal S, Rehana S, Lawrence D (2006) Unique type of isolated cardiac valvular amyloidosis. Journal of Cardiothoracic Surgery 25(1): 38 .

4. Rogers WR, Wittels B (1957) Extreme bilateral atriomegaly; Review of the literature and report of a case. Circulation 15(3): 434-441.

5. Gillmore JD, Wechalekar A, Bird J, et al. (2015) Guidelines on the diagnosis and investigation of AL amyloidosis. British Journal of Haematology 168: 207-218.

6. Ternacle J, Krapf L, Mohty D, et al. (2019) Aortic stenosis and cardiac amyloidosis: JACC review topic of the week. Journal of American College of Cardiology 74(21): 2638-2651.

7. Groves PH, Douglas-Jones AG, Hall RJ (1993) Amyloid, thrombosis, and acute myocardial infarction in association with a bicuspid aortic valve. Heart 70: 560-562.

8. Paul R S, Kush P P, Thomas A T, et al. (2020) Prevalence and outcome of dual aortic stenosis and cardiac amyloid pathology in patients referred for transcatheter aortic valve implantation. European Heart Journal 41: 1-10. 